\title{
How tongue strength exert influence on muscle strength and skeletal muscle of young adults.
}

\author{
Jong-Hwan Park ${ }^{1 \#}$, Eun-Seok Yeo ${ }^{2 \#}$, Bohyeon Kim¹, Seok-Hun Lee², Yoo-Chan Kwon ${ }^{3 *}$ \\ ${ }^{1}$ Health Behaviours and Disease Prevention Research Group, Institute of Convergence Bio-Health, Dong-A University, \\ Busan, Republic of Korea \\ ${ }^{2}$ Department of Taekwondo, Dong-Eui University, Busan, Republic of Korea \\ ${ }^{3}$ Department of Taekwondo, Dong-A University, Busan, Republic of Korea \\ \#These authors contributed equally to this work.
}

\begin{abstract}
The purpose of this study was to determine if an association exists between tongue strength and lower muscle strength, and skeletal muscle mass, in Taekwondo athletes. In this cross-sectional study, a total of 35 young adults aged $\geq 19$ (14 women and 21 men) gave their written informed consent to participate. A bio-impedance body composition analyser determined body fat percentage, skeletal muscle mass, and other body composition values. A hand-held dynamometer was used to evaluate quadriceps lower muscle strength. Tongue strength was assessed by measuring maximal tongue pressure against a tongue depressor connected to a strain gauge dynamometer. Partial correlation coefficients for tongue strength significantly correlated with skeletal muscle mass $(r=0.518, P=0.001)$, grip strength $(r=0.460, P=0.005)$, and lower muscle strength $(\mathrm{r}=\mathbf{0 . 4 8 7}, \mathrm{P}=\mathbf{0 . 0 0 3})$. However, tongue strength was inversely correlated with flexibility $(\mathrm{r}=-\mathbf{0 . 4 0 6}, \mathrm{P}=\mathbf{0 . 0 1 6})$. After adjusting for sex, tongue strength remained significantly correlated with skeletal muscle mass $(r=0.345, P=0.045)$, and lower muscle strength $(r=0.346, P=0.045)$. This crosssectional study is the first to report the association between tongue strength and grip strength, lower muscle strength, and skeletal muscle mass, in Taekwondo athletes. Our data suggests that the measurement of tongue strength may provide a new approach to determining muscle strength.
\end{abstract}

Keywords: Physical function, Skeletal muscle mass, Tongue strength

Accepted on November 13, 2017

\section{Introduction}

The tongue consists entirely of muscle and plays an essential role by exerting the maximum force required to propel food, liquid, secretions, and medicine within the oral cavity toward the oropharynx, and ultimately the oesophagus, during swallowing [1]. Moreover, tongue strength can be measured easily: There is wide variability in the responses of healthy individuals [2].

A decline in muscle strength may indicate fatigue, which is characterized by an acute reduction in the ability to exert muscle strength [3]. Age-related loss of muscle mass is associated with a decrease in muscle strength (sarcopenia), with this functional decrease appearing in striated muscle tissues. There is also involvement of the muscles that support swallowing, such as those found in the lips, tongue, and cheeks [4]. Previous studies have examined the effects of aging on tongue force and the consequent weakness in swallowing. A relationship between tongue lifting force and age has been reported; older individuals exert less tongue force during lifting than young adults [5].

Assessment of muscle strength is an important predictive factor, not only for neurological diseases or musculoskeletal disorders, but also for physical function. Lower extremity muscle strength is known as an important indicator of gait and physical function [6,7]. Some studies have examined the relationship between tongue strength and age, grip strength, jump height, and power in healthy older adults [8].

To the best of our knowledge, no previous studies have used an integrated approach to assess lower extremity muscle strength and tongue strength in Taekwondo athletes. Therefore, the purpose of this study was to examine whether an association exists between tongue strength, and lower muscle strength and skeletal muscle mass, in Taekwondo athletes.

\section{Materials and Methods}

A total of 35 young adults aged $\geq 19$ (14 women and 21 men) gave their written informed consent to participate in this study. 
Thirty-five elite Taekwondo athletes, with a minimum of 8 years competitive experience in the sport, voluntarily participated in this study. All participating athletes were black belt holders and classified in the national Division I category. Subjects were free of hypertension, dyslipidaemia, musculoskeletal injuries, and cardiovascular/metabolic disorders (Table 1).

Table 1. Baseline characteristics of the subjects.

\begin{tabular}{|c|c|}
\hline Gender, male/female & $21 / 14$ \\
\hline Age (years) & $20.9 \pm 0.9$ \\
\hline Body mass (kg) & $61.0 \pm 10.1$ \\
\hline Height (m) & $1.71 \pm 6.55$ \\
\hline BMI $\left(\mathrm{kg} / \mathrm{m}^{2}\right)$ & $20.7 \pm 2.5$ \\
\hline Body fat (\%) & $13.8 \pm 10.1$ \\
\hline SMM (kg) & $29.3 \pm 6.5$ \\
\hline Grip strength (kg) & $35.1 \pm 8.6$ \\
\hline Sit-up (frequency) & $28.4 \pm 7.0$ \\
\hline Flexibility (cm) & $18.9 \pm 8.5$ \\
\hline OLCE (sec) & $64.3 \pm 62.4$ \\
\hline Gait speed (sec) & $2.96 \pm 0.39$ \\
\hline LMS (Nm) & $201.6 \pm 30.4$ \\
\hline TS (Nm) & $3.71 \pm 0.98$ \\
\hline
\end{tabular}

Values are mean \pm SD; BMI: Body Mass Index; SMM: Skeletal Muscle Mass; OLCE: One Leg while Closing the Eyes; LMS: Lower Muscle Strength; TS: Tongue Strength

Body mass was measured to the nearest $0.05 \mathrm{~kg}$ using a digital scale (Inner-Scan 50; Tanita Co, Tokyo, Japan). Height was measured to the nearest $0.1 \mathrm{~cm}$ using a wall-mounted stadiometer (YS-OA; As One Co, Osaka, Japan). Body mass index (BMI) was calculated as weight in kilograms divided by the height in meters squared. A bio-impedance body composition analyser (Jawon Medical, Gyeongsan, Korea) determined the body fat percentage $(\% \mathrm{BF})$, skeletal muscle mass (SMM), and other body composition values [9].

Grip strength was measured using a dynamometer (Takei TKK 5401, Takei Scientific Instruments Co. Ltd., Tokyo, Japan), with the maximum strength (in $\mathrm{kg}$ ) over two attempts with the dominant hand being used. A sit-up was measured by maintaining a knee angle of 140 degrees while the subject lay on the mat with feet touching the floor, and the upper body rising to touch the knee with the elbow for 30-seconds. For balance measurement, the subject stood on one foot with their eyes closed while raising the other foot, and putting both hands on the waist. The time until balance was lost was measured twice at 0.1-second intervals, and the average time was recorded. A sit and reach flexibility-measuring tool (TKK 5103, Japan) was used to measure flexibility.
Spatiotemporal parameters of gait were assessed using a validated [10] wireless inertial sensing device (BTS Bioengineering, Italy) attached to the subject's waist using a semi-elastic belt, covering the L4-L5 intervertebral space, to acquire acceleration values for three anatomical axes: the anteroposterior, medial-lateral, and vertical axes. Subjects were asked to walk along an 8-m pathway at a self-selected speed as naturally as possible. The collected data was transmitted via Bluetooth to a PC and processed using dedicated software (BTS G-Walk).

A hand-held dynamometer (HDD) (JTECH Medical, Salt Lake City, UT, USA) was used to evaluate quadriceps lower muscle strength (LMS); the reliability of this method had been validated in another study [11]. In short, with each subject sitting, the knee joint was flexed from the maximum extension angle by $35^{\circ}$, after which an HDD was positioned and fixed on the ankle to allow for an isokinetic dynamometry recording of the isometric peak flexion forces (the $\mathrm{N}$ values) of both knee joints.

All measurements were made while the subjects were seated in a comfortable high-backed armchair. The tongue strength was assessed by measuring maximal tongue pressure against the tongue depressor connected to a strain gauge dynamometer (dynamometer of tongue muscle; Takei Ltd., Niigata, Japan). Peak value during 5-second maximal voluntary tongue protrusion was evaluated for each participant. The higher value of two consecutive measurements, spaced 2-minutes apart, was defined as the strength of genioglossus [12].

Data were analysed using the IBM SPSS Statistics version 22.0 software package for Windows (SPSS Inc., Chicago, IL, USA). Linear trends were assessed with the Kruskal-Wallis test (twotailed) for the median value in each category. Spearman correlation coefficients were calculated between tongue strength, and lower muscle strength and physical fitness parameters, respectively. Partial correlation coefficients were also calculated between tongue strength, and lower muscle strength and physical fitness parameters, respectively, with adjustment for sex. Statistical significance was accepted at the $5 \%$ level. Results are presented as means \pm standard deviation (SD).

\section{Results}

Thirty-five subjects (14 women and 21 men) were recruited. Characteristics of the subjects are presented in Table 1. Skeletal muscle mass of the subjects was $29.3 \pm 6.5 \mathrm{~kg}$. Lower muscle strength was $35.1 \pm 8.6 \mathrm{~kg}$, and tongue strength was $3.71 \pm 0.98 \mathrm{~kg}$.

Table 2 shows the partial correlation coefficients for tongue strength were significantly correlated with SMM ( $\mathrm{r}=0.518$, $\mathrm{P}=0.001)$, grip strength $(\mathrm{r}=0.460, \mathrm{P}==0.005)$, and LMS $(\mathrm{r}=0.487, \mathrm{P}=0.003)$. However, tongue strength was inversely correlated with flexibility $(\mathrm{r}=-0.406, \mathrm{P}=0.016)$. After adjusting 
for sex, tongue strength remained significantly correlated with SMM ( $\mathrm{r}=0.345, \mathrm{P}=0.045)$, and LMS $(\mathrm{r}=0.346, \mathrm{P}=0.045)$.

Table 2. Correlations between tongue strength and both body composition and physical fitness parameters.

\begin{tabular}{lll}
\hline Variable & Unadjusted & Adjusted \\
\hline BMI $\left(\mathrm{kg} / \mathrm{m}^{2}\right)$ & 0.254 & 0.268 \\
\hline Body fat (\%) & -0.240 & -0.001 \\
\hline SMM (kg) & $0.518^{* *}$ & $0.345^{*}$ \\
\hline Grip strength $(\mathrm{kg})$ & $0.460^{* *}$ & 0.236 \\
\hline Sit-up (frequency) & 0.253 & -0.071 \\
\hline Flexibility (cm) & $-0.406^{*}$ & -0.342 \\
\hline OLCE (sec) & -0.213 & 0.052 \\
\hline Gait speed (sec) & 0.011 & -0.001 \\
\hline LMS (Nm) & $0.487^{* *}$ & $0.346^{*}$ \\
\hline
\end{tabular}

Spearman correlations were used to calculate coefficients of correlation between vitamin D levels, and lipid and physical fitness parameters; Statistical analysis was conducted by using partial correlations, with adjustment for gender; Values are mean \pm SD. BMI: Body Mass Index; SMM: Skeletal Muscle Mass; OLCE: One Leg While Closing The Eyes; LMS: Lower Muscle Strength.

\section{Discussion}

To the best of our knowledge, this study is the first to report the relationship between tongue strength and skeletal muscle mass, in Taekwondo athletes. The main finding was a positive correlation between tongue strength and lower muscle strength and grip strength, suggesting that physically active young individuals, at least in our study population, have higher tongue strength.

In this study, we observed that tongue strength was more likely to be associated with grip strength in Taekwondo athletes. Although the association between tongue strength and grip strength in relation to primary sarcopenia has been reported in healthy adults [8], to our knowledge, this is the first study to reveal an association between tongue strength and grip strength, which may be utilized as a new measurement parameter in athletes. In addition, maximum tongue strength is higher in healthy young people than in healthy older people, while normal swallowing tongue pressure does not differ significantly in the two groups [13]. Thus, it seems reasonable to speculate that tongue strength partially reflects increased physical performance in young adults.

We also showed that tongue strength was positively correlated with lower muscle strength in Taekwondo athletes. This finding of an association between lower muscle strength and tongue strength shows the necessity for consideration of including both measurements when measuring physical function. Burkhead et al. [14] mentioned that swallowing is a sub-maximal muscular activity and that the muscular force generated to successfully complete the activity is much lesser than the maximal force that could be generated by the muscle involved. This could be explained thus; the positioning executions of this novel measurement are considered to rigorously activate the swallowing-related lower muscle strength and skeletal muscle mass.

Several study limitations are worth noting. Firstly, as the present study employed a cross-sectional research design, causal relationships could not be determined. Secondly, we did not use dual-energy x-ray absorptiometry or bioelectrical impedance analysis to measure muscle mass. Thirdly, because the measurements of tongue strength were taken only at the anterior tongue, the association with tongue strength at the posterior tongue is unclear. Finally, the sample size was small, which limited our ability to determine the significance of the results. Therefore, additional studies with larger sample sizes are required to determine the relationships observed in this study.

\section{Conclusions}

In conclusion, this cross-sectional study is the first to report an association between tongue strength, and grip strength, lower muscle strength, and skeletal muscle mass, in Taekwondo athletes. Our data suggests that the measurement of tongue strength may provide a new approach to determining muscle strength. It would be interesting to investigate whether tongue strength training interventions can lead to the improvement of muscle strength in athletes.

\section{Acknowledgements}

This work was supported by the Dong-A University research fund.

\section{References}

1. Youmans S, Stierwalt JAG. Measures of tongue function related to normal swallowing. Dysphagia 2006; 21:102111.

2. Utanohara Y, Hayashi R, Yoshikawa M, Yoshida M, Tsuga K, Akagawa Y. Standard values of maximum tongue pressure taken using newly developed disposable tongue pressure measurement device. Dysphagia 2008; 23: 286-290

3. Gandevia SC. Spinal and supraspinal factors in human muscle fatigue. Physiol Rev 2001; 81: 1725-1789.

4. Gingrich LL, Stierwalt JA, Hageman CF, Lapointe LL. Lingual propulsive pressures across consistencies generated by the anteromedian and posteromedian tongue by healthy young adults. J Speech Lang Hear Res 2012; 55: 960-972.

5. Clark HM, Solomon NP. Age and sex differences in orofacial strength. Dysphagia 2012; 27: 2-9

6. Bean JF, Kiely DK, Herman S, Leveille SG, Mizer K, Frontera WR, Fielding RA. The relationship between leg power and physical performance in mobility-limited older people. J Am Geriatr Soc 2002; 50: 461-467. 
7. Suzuki T, Bean JF, Fielding RA. Muscle power of the ankle flexors predicts functional performance in communitydwelling older women. J Am Geriatr Soc 2001; 49: 1161-1167.

8. Buehring B, Hind J, Fidler E, Krueger D, Binkley N, Robbins J. Tongue strength is associated with jumping mechanography performance and handgrip strength but not with classic functional tests in older adults. J Am Geriatr Soc 2013; 61: 418-422.

9. Völgyi E, Tylavsky FA, Lyytikäinen A, Suominen H, Alén M, Cheng S. Assessing body composition with DXA and bioimpedance: effects of obesity, physical activity, and age. Obesity 2008; 16: 700-705.

10. Bugané F, Benedetti MG, Casadio G, Attala S, Biagi F, Manca M, Leardini A. Estimation of spatial-temporal gait parameters in level walking based on a single accelerometer: Validation on normal subjects by standard gait analysis. Comput Methods Programs Biomed 2012; 108: $129-137$.

11. Kim WK, Kim DK, Seo KM, Kang SH. Reliability and validity of isometric knee extensor strength test with handheld dynamometer depending on its fixation: a pilot study. Ann Rehabil Med 2014; 38: 84-93.
12. Kanezaki M, Ogawa T, Izumi T. Tongue Protrusion Strength in Arousal State Is Predictive of the Airway Patency in Obstructive Sleep Apnea. Tohoku J Exp Med 2015; 236: 241-245.

13. Nicosia MA, Hind JA, Roecker EB, Carns M, Doyle J, Dengel GA, Robbins J. Age effects on the temporal evolution of isometric and swallowing pressure. J Gerontol A 2000; 55: M634-M640.

14. Burkhead LM, Sapienza CM, Rosenbek JC. Strengthtraining exercise in dysphagia rehabilitation: principles, procedures, and directions for future research. Dysphagia 2007; 22: 251-265.

\section{*Correspondence to}
Yoo-Chan Kwon
Department of Taekwondo
Dong-A University
Republic of Korea 